\title{
A Biofeedback Game with Physical Actions
}

\author{
Nagisa Munekata ${ }^{1}$, Teruhisa Nakamura ${ }^{2}$, Rei Tanaka ${ }^{2}$, \\ Yusuke Domon ${ }^{2}$, Fumihiko Nakamura ${ }^{1}$, and Hitoshi Matsubara ${ }^{2}$ \\ ${ }^{1}$ Hokkaido University, Sapporo, Japan \\ \{munekata@complex.eng, fumihiko-n@ec\}.hokudai.ac.jp \\ http://www.hokudai.ac.jp \\ ${ }^{2}$ Future University-Hakodate, Hakodate, Japan \\ \{b1007161, b1007216, b1007198, matsubar\}@fun.ac.jp \\ http://www.fun.ac.jp
}

\begin{abstract}
We developed a biofeedback game in which players can take other physical actions besides simply "relaxing". We used the skin conductance response for sensing a player's surge of excitement and penalized players when they did not attack enemies in situations because they were not calm enough to meet the biofeedback threshold. We conducted a subjective experiment to to see whether people found the game enjoyable. Most participants felt the game was enjoyable.
\end{abstract}

Keywords: biofeedback, phisical actions, skin conductance response.

\section{Introduction}

Various forms of entertainment with biofeedback (BF) are being developed. This new trend is exemplified by Nintendo Ltd's announcement that it was releasing a "Wii vitality sensor" that would measure players' heart beat and other biological signals, as a peripheral device for its Wii game platform [1]. A game with BF has an unusual property whereby the player's mental concentration is the key to victory or defeat 23. The most popular video game series with $\mathrm{BF}$ is "The Journey to Wild Divine" [4. The blurb on this game reads "Daily mind and body practice for healthy living," and the game uses BF to make the user learn proper breathing and meditation techniques. In such games, the player completes game stages when he or she strives to relax through mental concentration. Most theorists endorse the view that emotions comprise three components: subjective experience (e.g., feeling joyous), expressive behavior (e.g., smiling), and a physiological component (e.g., sympathetic arousal); others add motivational state or action tendency and/or cognitive processing to that list [5]. However, most $\mathrm{BF}$ games have been regarded as merely instruments for the user's health. That is, they are unsuitable as entertainment because if the user falls into a relaxed state while playing a video game they will become bored with it and eventually quit the game. Thus, BF has been viewed as a technology by which the user's mind can perceives the body's state in order to control it. BF is known for its sedating effect; it reduces stress or excitement and promotes relaxation. 


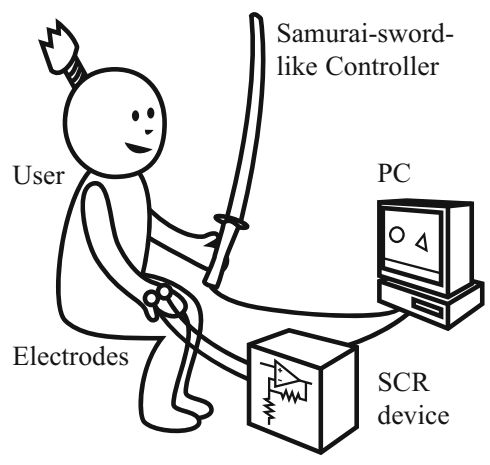

Fig. 1. System overview

Moreover, a common characteristic of BF games is that the player succeeds by keeping relaxed. Such a goal is not especially good for entertainment content, and thus far, BF's application to games has been limited. In contrast to the above passive uses, we have developed a number of BF games in users can be excited and agitated 6 6/78. These games have the purpose of inducing "outof-control" emotions such as the feeling of being on a tightrope. Our previous studies were dedicated to exploring the entertainment possibilities of BF. Many games, including those developed by us, however, require a player to relax in a sitting posture and maintain a resting state. The inputs to these games consist of only the emotional expressions mediated by BF as well as pushing of controller buttons and arrow keys. In this study, therefore, we developed a BF in which the user not only has to relax but also has to execute physical motions. Specifically, the user has to shake the hand controller to have an effect on the game. we thought the introduction of physical motions as a second form of input would excite user and enhance the quality of entertainment despite that user should stay relaxed. We evaluated this game in a subjective experiment that assessed the users' feelings of excitement.

\section{System Configuration}

The electrodes are placed on the user's hand to measures change in his or her skin conductance response (SCR) (see Fig 1). The changes sensed by the SCR values reflect the user's excitement regarding the gameplay. We made the game harder for the player to win if these changes are large. Moreover, we made a samurai-sword-like controller as the way of inputting physical actions. The user inputs an action by shaking the controller strongly. The idea is for the user to hit "enemies" in the game by using this controller. The elements of the game system are detailed below. 

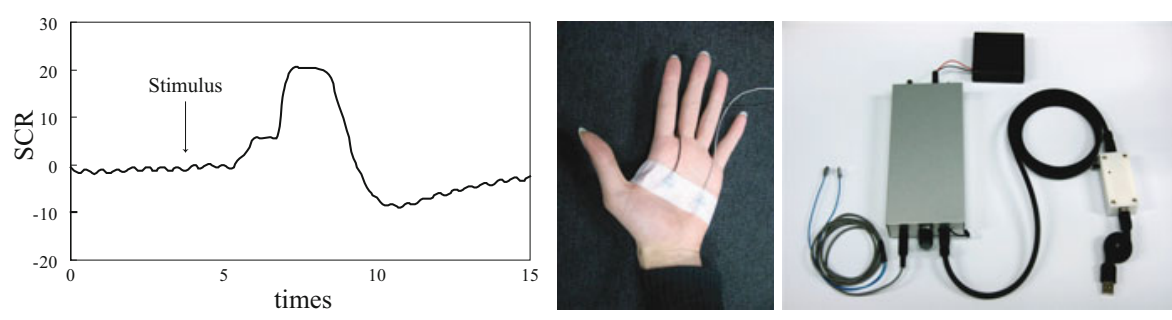

Fig. 2. Graph of SCR and device

\subsection{Skin Conductance Response}

Electrical signals from a living body have been used for medical diagnosis and treatment and in lie detectors used in police questioning [9]. The biological signal used in the lie detector is the skin conductance response (SCR); the conductance on the skin surface changes as a result of sweating induced by mental agitation, surprise, and excitement [10. We have little awareness of the physiological functions of our own body because most physiological functions are involuntary, and therefore uncontrollable. The SCR is a typical example in this regard. No one is aware of the minute amounts of sweat that well up during mild mental agitation. Figure 2 shows an SCR graph, the measuring device and a user's hand with electrodes attached to it.

\subsection{Samurai-Sword-Like Controller}

The samurai-sword-like controller is a sword shaped toy with a built-in triaccelerator. The system converts analog data from the tri-accelerator (AEKXP84) into digital data by using a microprocessor (PIC12F675), and it is connected to a PC through a USB conversion module (AE-UM232R) and USB mini cable. We made a sword hilt with a built-in base $(2 \times 6 \mathrm{~cm})$ that was soldered to the tri-accelerator, microprocessor, and USB conversion module. The USB mini cable can be unspooled from a small hole in the hilt and connected to the PC. When the user shakes this controller vigorously (acceleration: more than $0.20 \mathrm{~m} / \mathrm{s}^{2}$ ), he or she can hit enemies in the game. We set the standard of "shaking vigorously" to be the minimum acceleration measured during a test in which ten persons shook the controller vigorously.

\subsection{Story of the Game}

We chose a "fly swatter" game in which the player hits and kills flies. In this game, the user must restrain his or her excitement and attack with physical movements. Needless to say, fly swatting is not easy in real life. If you swat too much, the flies will notice the danger and fly away. As in real life, the player in the game must relax in order to succeed and he or she must attack quickly when the chance comes. There is a strong resemblance between the intended attitude 


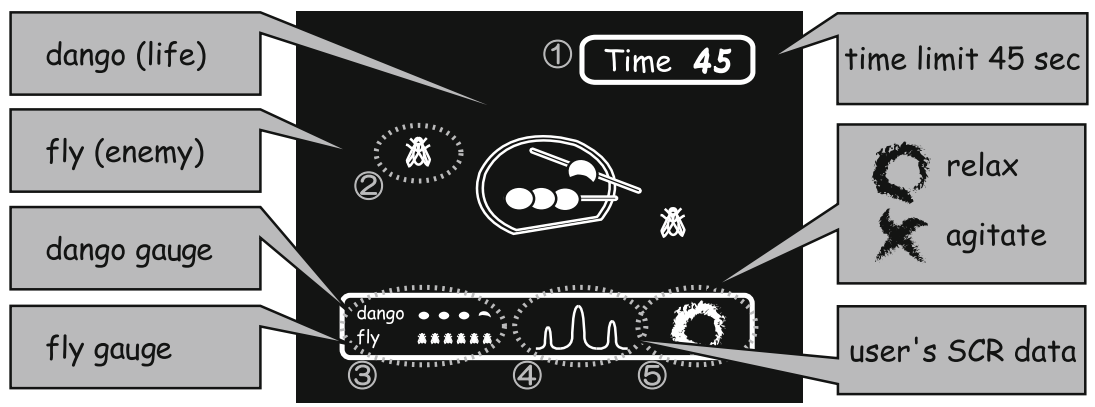

Fig. 3. Display of the game

and the purpose of this game, and that is why we thought the game would be a good BF application.

The flow of this game is as follows. First, the user watches a movie introducing the story behind the game. In this movie, a samurai is resting in a teahouse and eating dangos. (A dango is a Japanese dumpling made from rice flour.) A samurai finds flies on his dangos. This makes him angry and he tries to swat them with his sword. After the user finishes watching this movie, the game begins and the user attempts to hit the flies with his or her samurai-sword-like controller.

\subsection{Game Rules}

Figure 3 shows (1) the time limit, (2) flies, (3) dango gauge and fly gauge, (4) SCR graph, and (5) state signs of attack (agitating: $\mathcal{X}$, not agitating: 0 ). (5) depends on "user's agitation" level as determined by the SCR value.

A user tries to hit flies on the screen until the time limit (45 seconds) expires. When the SCR values exceed the threshold value (70), the user is deemed to be agitated, and the " $\boldsymbol{X}$ " graphic is displayed at position "(5)" on the screen. On the other hand, when the SCR does not exceed the threshold, the user is deemed to be relaxed and "O" graphic appears. The user can hit flies whenever he or she wants. However, if the user tries to hit them when the " $\boldsymbol{X}$ " mark is displayed, the attack fails and the fly steals a dango. Thus, the game progresses poorly if the user attacks too often without paying attention to the BF and relaxing. In addition because of the time limit, a player cannot succeed without attacking quickly. The threshold value was taken from the results of a previous study $67 / 8]$. Three flies on average appear during five seconds, and their appearance within is uniformly random. After the game is over, the user's samurai level is displayed. The score is calculated from the evaluation value of each parameter in Fig. 4. The purpose of showing the "level of samurai" is to enhance the quality of entertainment because it is easy to compare to one's own level with others. The parameters are described below.

- Remaining flies: the remaining number of flies in game (fly gauge): default value - twenty

- Dangos remaining: the remaining number of dangos in the game (dango gauge): default value - four 


\begin{tabular}{llr}
\hline & \multicolumn{1}{c}{ User's state and judgement } & Point \\
\hline escapeFly & A fly is escaped from the display & -1 \\
\hline overThreshold & User's SCR is over the threshold & -5 \\
\hline Remaining dangos & User attack on a fly when user's SCR is over the threshold & -10 \\
\hline Display smached & Despite there are no flies, user attack on them three times & -10 \\
\hline brokenTeahouse & “crackDisplay” is occurred four times & -30 \\
\hline gameClear & All flies are exterminated & +30 \\
\hline
\end{tabular}

Fig. 4. Detected user behaviors and corresponding points added or subtracted

We determine the "level of samurai" from the user's actions (see Fig. 4). Points are deducted and awarded as follows. flyEscaped means that the user attacks fly when the ' $\boldsymbol{X}$ " mark is displayed, the attack fails and the fly steals a dango. overThreshold means the user was excited and the SCR exceeded the threshold. stolenDango means that user attacked despite being overThreshold. At the same time, Remaining dangos is decremented. The game is over when Remaining dangos reaches zero. Display smashed means the user attacked even though flies were not on the screen. Such an act is considered very inappropriate of a samurai, and it thus entails a penalty of -30 points. brokenTeahouse means Display smashed occurred four times. If the user breaks the teahouse, the game is over and the user did not successfully complete the game. gameClear means that Remaining flies is zero and all of the flies were swatted. It entails a 30 point bonus.

We made 100 points the highest score and 0 the lowest. Moreover, this game is completed successfully when Remaining flies(fly gauge) reaches zero. The game finishes unsuccessfully when the Time limit is reached, Remaining dangos is zero (all of dangos were stolen by flies) or the teahouse becomes broken brokenTeahouse.

\section{Experiment}

The purpose of this experiment was to determine whether users would enjoy playing this BF game. So we investigated how BF affected the user in this game by comparing the SCR values with and without BF.

\subsection{Experimental Method}

Nine university students (eight men and one woman: 20-23 years old) participated in the experiment. As the dummy indicator, the average of randomly chosen SCR values of all participants was displayed as the SCR graph (Fig 3 . (4), and this average was used to determine the state signals of attack ( $\boldsymbol{X}$ or O) (Fig 3 : (5)). Before starting the experiment, participants watched a movie (about three minutes long) on how to play. Participants played the game with BF (phase 1) and without BF (phase 2) for three turns in the same order. They 


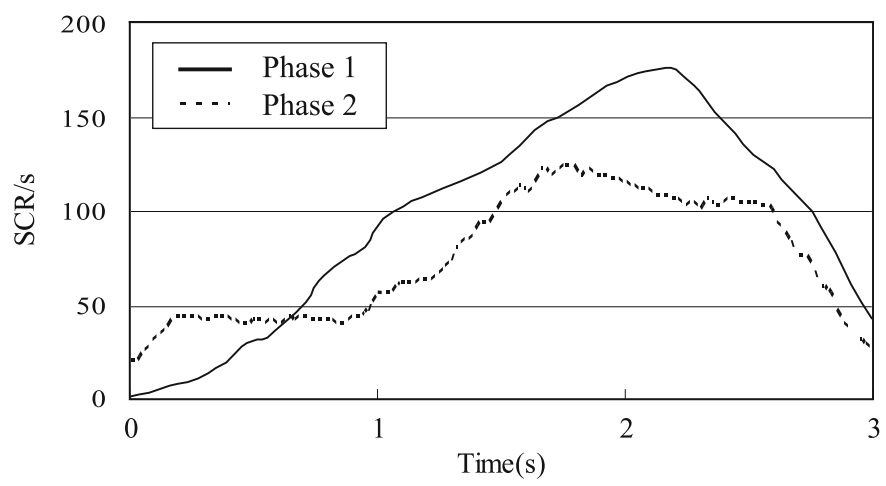

Fig. 5. Experimental results

were not informed of the distinction between these games. At the end of the experiment, the players were interviewed about whether they found the game enjoyable. Participants were randomly assigned to the following two groups:

- Group A: Participants undertook three trials for phase 1 and three trials for phase 2, and

- Group B: Participants undertook trial reversed phase order, i.e., phase 2 then phase 1.

\section{$3.2 \quad$ Results}

The $\log$ data of phase 1 and phase 2 were analyzed (Fig 5 ). In Fig. 5. SCR/s was defined as the SCR values per unit time and the extracted range was between the moment that the participant attempted to hit the fly and three seconds later. The results of a dispersion analysis showed significant differences $(F(1,202)=$ $26.20, p<0.01)$ between group A and group B. This indicated the game with BF (phase 1) could make the user significantly excited more than the game without BF (phase 2.) Additionally, none of the participants notice that different games were played or that phase 1 was different from phase 2 .

\subsection{Discussion}

The participants' excitement during phase 2 was significantly lower than in phase 1. This indicated that not only the SCR graph (Fig 3 : (4) ) and the state signals of attack ( $\boldsymbol{X}$ or $\mathbf{O})(\mathrm{Fig}, 3:$ (5) ) on the game screen increased excitement and agitation but also that the user's SCR value was the most important element contributing to excitement. Although the tests were conducted only six times, the results seem to indicate that had they played this game more, it would become easier for them to distinguish the phases 1. Participants affected their SCR values naturally without feigning even though they did not notice these distinctions. Therefore, we can conclude that the system exploited the characteristics of BF effectively to raise the users' level of excitement. 
Let us consider the meaning of the SCR graph and the state signals of attack (Fig[3). People usually go about their daily business with few chances to assess their behavior on the basis of physiological signals. However, they may become partially aware of certain states of the body and mind without using equipment. For example, a rapid heartbeat may signal one of many states and user might try to calm his or herself by breathing deeply. Other people might observe that sweating from palms indicates a state of anxious suspense. These signals are natural responses, and there are cases in which the reason for them can be revealed by tracing memories even when the reason for physical changes cannot be quickly recognized. People always try to take the optimum actions to fit the situation by observing and altering their own states. Participants who played the game in this study also tried to make their state ideal for the game, for instance, by deep breathing, humming or easing the tension in their shoulders. However, these actions tended to increase the participants' stress or agitation level.

In fact, it is difficult to recreate the critical conditions under which the user would feel themselves in danger in a game. However, as evidenced by the significant autonomic nervous system activity of palms sweating and the participants' responses, we believe that a game involving awareness of physical signals in the form of biofeedback would have good entertainment value.

\section{Conclusion}

We created a new BF game that stimulates the player's sense of agitation. This game requires the player not only to relax but also to perform physical actions. Previous BF games have had elements whereby the user's mental concentration makes the situation advantageous and his or her agitation makes the situation disadvantageous. We added a new element: i.e., an attacking action that involves the player shaking a controller with one hand. We experimented with this BF game to examine whether the players would feel more agitation when the BF was used and in turn feel more entertained.

The experimental result showed that the BF game was entertaining and that the individual variation in the SCR values had very little influence on the game's outcome. In addition, the experimental results showed that the sources of the user's agitation came from not only making attacks but also from a situation in which he or she had to inhibit their level of agitation. Moreover, we found that feeding back the user's own SCR values in real-time helped to increase their level of agitation. Additionally, the participants in the experiment intuitively understood that the fly could not be hit when they themselves were agitated. The clear influence of the SCR values on the game reinforced the idea that to make the attack succeed, calm judgment is needed.

In past $\mathrm{BF}$ games, the influence of individual variations was a problem that needed to be solved. In this game, the problem was solved by involving BF in the user's attack behavior. Users showing a wide range of individual variations in SCR found the BF game to be entertaining. We added a second input method (physical action) that past BF games did not have. This input method agitated 
the user and improved the entertainment value, without deviating from the concept of a BF game. Until now, an entertainment application of BF had been assumed to be problematic. Our experimental results, in contrast, indicate that $\mathrm{BF}$ may be used for entertainment purposes in the future.

We will further the possibilities of BF based on this game and the experimental results obtained in this study. We wish to clarify the prospects of using BF in various fields, by developing an affective system that generates appropriate events for the user while monitoring his or her physiological state.

\section{References}

1. Nintendo Co., Ltd., http://www.nintendo.co.jp/index.html

2. Breathing Space, Media Lab Europe (2003)

3. Hand-held Doctor. MIT Media Laboratory (1997)

4. The Wild Divine Project, http://www.wilddivine.com

5. Ravaja, N., Saari, T., Laarni, J., Kallinen, K., Salminen, M., Holopainen, J., Jarvinen, A.: The Psychophysiology of Video Gaming: Phasic Emotional Responses to Game Events. In: Proceedings of DiGRA 2005 Conference: Changing Views-Worlds in Play, 16-20, 2005, pp. 1-13 (2005)

6. Sakurazawa, S., Yoshida, N., Munekata, N., Omi, A., Takeshima, H., Koto, H., Gentsu, K., Kimura, K., Kawamura, K., Miyamoto, M., Arima, R., Mori, T., Sekiya, T., Furukawa, T., Hashimoto, Y., Numata, H., Akita, J., Tsukahara, Y.: A Computer Game Using Galvanic Skin Response. In: 2nd IFIP International Conference on Entertainment Computing, pp. 31-35. Carnegie Mellon University, Pittsburgh (2003)

7. Sakurazawa, S., Yoshida, N., Munekata, N., Tsukahara, Y., Matsubara, H.: Entertainment Feature of the Computer Game Using a Skin Conductance Response. In: ACM SIGCHI International Conference on Advances in Computer Entertainment Technology, pp. 181-186. ACM Press, New York (2004)

8. Munekata, N., Yoshida, N., Sakurazawa, S., Tsukahara, Y., Matsubara, H.: Design of positive biofeedback using a robot's behaviors as motion media. In: Harper, R., Rauterberg, M., Combetto, M. (eds.) ICEC 2006. LNCS, vol. 4161, pp. 340-349. Springer, Heidelberg (2006)

9. Geddes, L.A.: History of the Polygraph, an Instrument for the Detection of Deception. Bio-med. Eng. 8, 154-156 (1973)

10. Gross, J.J., Levenson, R.W.: Hiding feeling, the acute effects of inhibiting negative and positive emotion. Journal of Abnormal Psychology 106, 95-103 (1997) 